\title{
The Representation of a Multimedia Franchise as a Single Entity: Contrasting Existing Bibliographic Entities With Web-Based Superwork Portrayals
}

\author{
Senan Kiryakos and Shigeo Sugimoto \\ Graduate School of Library, Information and Media Studies, \\ University of Tsukuba, Japan \\ senank@gmail.com; sugimoto@slis.tsukuba.ac.jp
}

\begin{abstract}
Background. Multimedia franchises may have a single origin, but over time develop into a network of related creative works in various media formats such as film, novels, animation, and video games. A single entity to represent a whole franchise is often utilized on the Web, but the ability for existing bibliographic models to represent this entity, which we refer to as the Superwork, is unclear.

Objective. This research sought to determine whether entities representing multimedia franchises and the relationships they contain can be accurately portrayed using current models and, if not, how a new entity can be differentiated from the entities in the existing models. It also examines the role of existing franchise-level concepts on the Web and their ability to define properties and boundaries.

Methods. We contrasted the franchise-level concept against official documentation and past research into similar entities (i.e. FRBR Work and FRBRoo Complex Work). A mapping and analysis of franchise articles on Wikipedia was performed to identify user-created boundaries and relationships between related instances.

Results. The analysis revealed that the concept of a singular entity for a multimedia franchise was not the intended use for existing bibliographic entities, and that users will create such entities to collocate resources belonging to a single franchise. The Wikipedia mapping showed how and where users established relationships between works and media types, and thus what entities the Superwork should be directly connected to.

Contribution. This study builds upon past bibliographic family and transmedia research, and suggests that more effort should be put towards examining existing collocation activities of users.
\end{abstract}

\section{INTRODUCTION}

As worldwide interest in previously niche media forms such as video games, manga and comic books, animation, etc. has grown tremendously, the phenomenon referred to as media franchise, transmedia, media mix, and other terms referring to the spreading of a single franchise over multiple media, has also greatly increased, with large multimedia franchises now existing across dozens of formats. Popular examples of these franchises include Pokémon, Star Wars, Ghost in the Shell, Tolkein's Middle-earth, the Marvel Cinematic Universe, and numerous others. These creative works typically began as an instance of a 
single medium. As a work expands to become a multimedia franchise, the association between the name of a franchise and a single medium tends to weaken, even if a particular format or media type is dominant. Henry Jenkins (2003) discussed this in his exploration of transmedia storytelling, claiming that a franchise such as Pokémon "unfolds across games, television programs, films, and books, with no media privileged over any other" (p. 2). This means that over time, a dynamic resource such as a multimedia franchise begins to form a unique entity that, while not a single creative work, refers to a network of related creative works and the set of concepts that they embody.

The existence of this umbrella entity raises several interesting questions, such as what its properties and boundaries are; how, if at all, a franchise entity differs from existing entities in various bibliographic models that represent creative works or concepts; and how similar entities are currently portrayed and utilized by users. In seeking to address these questions, the research presented here proposes a bibliographic entity, referred to as the Superwork, which is meant to represent an entire multimedia franchise. These questions are approached in the following ways:

(1) Investigating related research: A small number of past studies have examined issues similar to those raised in this paper. In investigating bibliographic family or transmedia concepts, past research has attempted to clarify both what types of works fall under the concept, and whether existing bibliographic entities are sufficient to portray them. Some studies concluded that entities such as the Functional Requirements for Bibliographic Records (FRBR) Work or Object Oriented FRBR (FRBRoo) Complex Work are capable. However, most of these studies use examples that more often resemble single creative works. Therefore, we examined the findings of these studies to determine their applicability to a whole multimedia franchise. The results indicate that the works discussed in past literature typically describe a work that is different from a franchise, and that FRBR 00 's Complex Work seem capable of describing franchises adequately. The lack of franchise-level research, however, led to our closer examination of Complex Work to determine its suitability for franchise representation.

(2) Examining existing bibliographic entities: Though existing bibliographic and other data models contain entities meant to represent creative works, often with fluid boundaries, it is unclear whether these are capable of, intended for, or ideal for describing an entity as broad as a franchise. Past research has suggested the FRBR Work and FRBR Oo $_{\text {Complex }}$ Work as entities that, if not already meant to describe a Superwork-like entity, are capable of doing so. To more explicitly differentiate them from Superwork, we closely examined Work and Complex Work using both past research and official documentation. We found that the examples used in the documentation, the supported relationship types, and other facets of these entities imply an intended use that is not equivalent to portraying franchise-level entities, and that Superwork is distinct enough to justify further research. Lastly, due to suggestions that a Superwork may represent a specific concept or topic rather than group of creative works, we examined how Superwork differs from and relates to topic.

(3) Portraying and characterizing Superwork: Based on the differentiation of Superwork from existing entities described in (2), we established some properties of Superwork that indicate what a Superwork is and is not. We conducted an examination of the specific facets and boundaries of Superwork using existing literature and Web portrayals of franchise relationship networks. A series of connected Wikipedia articles representing a 
single multimedia franchise was mapped and surveyed, which resulted in some clarification in understanding how users set existing boundaries for a collocated franchise entity, and how and where relationships between entities in the franchise are made. Importantly, these findings helped to resolve some outstanding issues that arose in past Superwork and transmedia research, though some new questions were also raised, which will help guide future research.

Thus, the first objective of the research presented in this paper compares the types of creative works discussed in past literature to the concept of multimedia franchise. The comparison revealed that the multimedia franchise concept was indeed distinct, as past literature tended to focus on either single creative works with closely related derivatives and adaptations, or a series of works that fell under the umbrella of a franchise, rather than the whole multimedia franchise which is the focus of this study. The paper then moves on to an analysis of related bibliographic entities and their suitability in representing a multimedia franchise, seeking to properly differentiate Superwork from FRBR Work and FRBR Complex Work, as well as the traditional subject entity. This is followed by visualization and exploration of single-entity descriptions of multimedia franchises on the Web, which served as user-contributed evidence for further developing the concept of franchise-level entity.

\section{LITERATURE REVIEW}

Though the idea of a franchise-level entity for use with large multimedia franchises has received little research, studies of similar bibliographic entities has been performed, often in the context of library collections and cataloguing. Nevertheless, past research on how to organize networks of related materials and the representation of that network as a single entity or in a single accessible location has been important in providing a basis for applying similar ideas to multimedia franchises. Such research includes examinations of how to develop Superwork-like entities (Carlyle, 1996; 1999; Smiraglia, 2007; Smiraglia, Riva, \& Žumer, 2014; Svenonius, 2000), studies on whole-part relationships between different bibliographic entities (Niu, 2013), establishing bibliographic families and instantiation networks based on related works (Leazer \& Smiraglia, 1999; Wilson, 1968), and visualizations of identity networks (Leazer \& Furner, 1999). More practical studies have attempted to implement bibliographic families or FRBR-based related work principles in navigational systems. The effects on user experience have been looked at by Ercegovac (2006) who found that creating links between entities led to a better grouping of those entities, with enhanced search result navigation and resource discovery and use. Merčun, Žumer, and Aalberg (2012 \& 2017) developed the FrbrVis prototype to create a hierarchical visualization of complex work sets, and found that visual displays of these networks were useful in exploring hierarchical and horizonal relationships among them.

As most of the existing research has focused on the library catalogue and its materials, its applicability to multimedia works and franchises is limited. Using a random sampling of 996 WorldCat records, Bennet, Lavoie, and O'Neil (2003) found that only $25 \%$ of works existed as multiple manifestations. Thus, as multimedia franchises by definition span multiple media formats, direct comparisons between models and solutions aimed primarily at the library catalogue or monographs should be done with caution. Teixeira (2012) argued that hierarchical representations such as FRBR have increased benefits for certain types of works such as serial collections or works that exist across different publishers, editions, and, importantly, media. While this argument remains true for works of multimedia, the suitability of the specific models for portraying certain types of relationship commonly found in 
multimedia franchises is questionable. The inadequacies of existing bibliographic models when applied to more niche formats and media are a common theme in some research on entities in popular culture. Studies that have focused on video games and anime (Jett, Sacchi, Lee, \& Clarke, 2016; Lee, Shim, \& Jett 2015) have found that models such as FRBR are unable to adequately portray the complex relationships between these resources and also fail to provide a level of description that sufficiently addresses user requirements. These studies emphasized the need for more detailed metadata to describe features such as genre, as these are deemed by users to be vital for search and discovery. Lee, Shim, and Jett (2015) performed a user study of fans of Japanese anime and noted the need for more descriptive metadata, including genre, art style and character types. Similar findings were reported by Kiryakos and Sugimoto (2015) and Kiryakos, Sugimoto, Nagamori, and Mihara (2016), who found that the incorporation of data from fan pages or other hobbyist Web resources, which were at different levels of granularity, could better meet the level of detail that users of popular culture materials required. Though these latter studies did not directly discuss the need for a franchise-level entity, the attempt to meet user needs for data at different granularity levels yielded some descriptive metadata that are more accurately attributed to the franchise as a whole, rather than a single series or media type. This highlights the need to establish an entity representing the franchise.

Related multimedia research exists as investigations into the transmedia phenomenon. Branch et al. (2017) developed an ontological model for describing transmedia fictional worlds for better knowledge organization and search and retrieval by end users. Though the model and its findings focused mainly on the contents of the transmedia franchises, their domain analysis revealed that in attempting to include fan-fiction and other non-licensed works, which are quite popular among most large multimedia franchises, "establishing relationships from these variants to the licensed elements" was a challenge (p. 2777). These fan works pose a similar challenge in the creation of a Superwork entity, particularly when attempting to define what types of fan works are included - if they are to be included at allin the range of works that a Superwork represents.

Additional past transmedia works (Jenkins, 2003; Dena, 2009; Giovagnoli, 2011) have thoroughly defined and explored the phenomenon, and have been vital in helping shape what the boundaries of a franchise may be, and thus what particular characteristics make a work suitable for inclusion in the Superwork umbrella. Though non-transmedia works have also discussed this issue in trying to define the Superwork or similar entity (e.g., Svenonius' (2000) notion that the Superwork include commentaries, references, and derivatives), the multimedia nature of the transmedia phenomenon is an important feature that should be examined separately from literature-centric research.

Ana Vukadin's (2014) work Bits and Pieces of Information: Bibliographic Modeling of Transmedia looked at modeling of transmedia works and, like the research presented here,

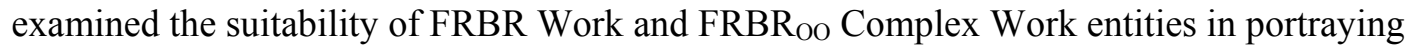
relationships in transmedia works. Vukadin did not argue for a singular franchise-level Superwork entity, but instead looked at the capability of Work and Complex Work to portray various relationships in transmedia. She found that though the Work entity was conceptual enough to encompass the overarching concept that individual works connected to a Superwork may share (IFLA Study Group on the Functional Requirements for Bibliographic Records, 2006, pp. 17, 26-27), the fundamental whole/part relationships in transmedia works could not be properly represented. Instead, "FRBRoo seems to offer more precise definitions for this purpose" using the Complex Work entity (Vukadin, 2014, p. 296). She also argued 
that the FRBRoo Expression Fragment subclass might be used to describe shared assets such as characters, settings and footage across transmedia works.

Some clarification on the term transmedia may be helpful here, as the meaning of the term differed between authors. The lack of uniformity in the use of the term appears to be centered on whether one considers that adaptations into different mediums constitute a work of transmedia, or whether transmedia requires a single narrative to be spread across multiple media. Vukadin (2014) stated that while adaptations use each medium to create a unique experience and different interpretation of a work, they "mostly transfer the same content from one medium to another" whereas transmedia "delivers unique pieces of content in each of the media channels, seeking to synchronize them and integrate them into a complex, yet coherent narrative structure" (p. 298). Branch et al. (2017) said that transmedia storytelling is "elements of a media property that advance the narrative" and that this differs from "elements that are simply brand expressions" (p. 2772). Giovagnoli (2011) stated that transmedia is a narrative form that shares "the same elements (plots, characters, atmospheres...) but that change depending on the publishing platform through which they are released" (p. 12). In referring to Jenkins, Dena (2009) "argues that practitioners should provide unique story information in each media. But when this happens fans of a fictional world are splintered into groups according to whether they are literate in the various media" (p. 160). Jenkins (2003) wrote, however, that ideally, each medium in transmedia storytelling "does what it does best" and that "each franchise entry needs to be self-contained enough to enable autonomous consumption. That is, you don't need to have seen the film to enjoy the game and vice-versa" (p. 4). This appears to argue that each medium exploits its strengths to tell an adapted story rather than a prescription that each should provide unique story information. Jenkins (2014) did also say that "consumers must assume the role of hunters and gatherers, chasing down bits of the story across media channels" to fully experience a fictional world (p. 21). Regarding adaptation, Dena stated that it is a central part of transmedia. Vukadin (2014) wrote that the TV series Game of Thrones "can be viewed as a new franchise expanding strategy rather than a mere TV adaptation of George R. R. Martin's novels" (p. 298). Though a formal clarification will not be attempted in this paper, any use of the term transmedia in this paper will refer to a franchise adapted into multiple media, with each adaptation able to exist on its own (i.e. analogous to the franchise-level Superwork entity), with transmedia storytelling being used for a single narrative spread across multiple media, requiring consumption of media on multiple formats for total coherence. This same distinction can be seen elsewhere (e.g., https://en.wikipedia.org/wiki/Transmedia_storytelling) and allows for both ideas to be expressed with hopefully less confusion.

An important piece of past research for this study was Tallerås, Dahl, and Pharo's (2018) study "User conceptualizations of derivative relationships in the bibliographic universe", which examined how users conceptualize and map relationships between related resources. Based on the title pages of play scripts, DVD covers of film adaptations, and CD covers of soundtracks of Peer Gynt and Romeo and Juliet, users were asked to map the relationships between these resources and apply attributes to each. The majority of the produced concept maps (62/99 or 63\%) featured a user-created "central node" that connected some or all of the three materials and featured attributes such as persons of responsibility, while attaching attributes such as date of publication to the document nodes. As the central node featured persons of responsibility (e.g., Shakespeare) that were not necessarily responsible for all of the documents attached to that node (e.g., the music $\mathrm{CD}$ ), the authors stated that "the central node cannot entirely be interpreted as an FRBR work" but instead it is "plausible to interpret the central node as an even more abstract collocating device" and "the 
central node more resembles a Superwork entity" (p. 911). The finding that "users seem to prefer multi-entity conceptualizations including a Superwork entity" (p. 912) emphasize the need to explore the development of entities representing multiple types of conceptual abstractions, namely the Superwork and fictional world.

Other niche-media focused Superwork research has been performed by McDonough, Kirschenbaum, Reside, Fraistat, and Jerz (2010), who found that in attempting to model computer video games, the number of related instances that can exist and the tendency for this network to continue to grow over time provided a "compelling justification for the notion of a Superwork" (p. 31). This notion of the relationship network growing over time provides support for the creation of a franchise-level entity, as Lee and Jacob (2011) found that the explicit relationships prescribed by FRBR "might be too rigid to support the flexibility necessary for describing dynamic resources" (p. 18). Additionally, McDonough et al. (2010) found that the ability to collocate many of these resources was important for users, and that the "application of a Superwork entity may be the simplest means of enabling users' preferred mode of searching" (p. 31). Kiryakos et al. (2017) performed preliminary work on exploring the relationships between related entities in a multimedia franchise and the inability for existing entities to properly describe them. This work was continued in Lee et al. (2018) with the researchers contrasting the Superwork concept with existing entities and attempting to define its properties and characteristics. The concept of the Superwork is similar to the one presented here, and this paper continues the work put forth in these previous two studies.

This review of past literature lays the foundation for addressing the concerns raised in the introduction: first, previous research looking at franchise-level entities has been conducted, though much of it focused on the library catalogue and library materials; second, the nature of multimedia franchises and dynamic resources has been shown to be very distinct from traditional library materials, and that alternative models and franchise-level entities can enable the portrayal of an entity in the way that users conceptualize them; and lastly, while research on the capability of existing entities to portray the relationships in multimedia

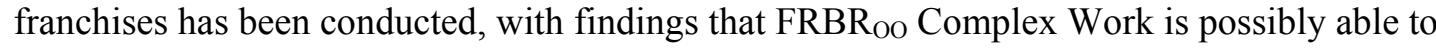
do so, a thorough comparison of these entities to existing portrayals of franchise-level entities, specifically from the Web, has yet to be undertaken.

\section{DIFFERENTIATING THE SUPERWORK FROM RELATED ENTITIES}

In this paper, a Superwork is meant to represent a franchise in its totality, free of single series or medium-specific context. In its most basic form, a Superwork can be seen as an entity that is the center of a number of whole/part relationships. Though a Superwork does not refer to a single instantiation or creative work, and is thus a conceptual entity, it does refer to a network of related works and the concepts that these works represent. The way these works are related may be through authorship, a shared universe or characters, a unifying fictional world, a brand, or other characteristics. As existing bibliographic models such as FRBR and FRBRoO include entities meant to represent the abstract, conceptual essence of creative works, it is worth investigating the capability of these entities to represent a franchise as a Superwork.

\section{Superwork versus FRBR's Work}

The FRBR Work entity is defined as "a distinct intellectual or artistic creation" (IFLA, 2006, p. 17) - a definition vague enough to be open to interpretation and to allow some fluidity when determining whether a certain instance is a Work (see Zhang and Salaba, 2012). While the FRBR documentation examples do not use Work to refer to an entity as broad as an entire 
multimedia franchise, some researchers have understood the definition as supporting the capability to do so. Svenonius (2000, p. 35) differentiated between the work as "The set of all documents sharing essentially the same information" and the Superwork as "The set of all documents descended from a common origin." Smiraglia (2007, p. 79) argued that the idea of Superwork and FRBR Work are the same (although, he also discussed a similar concept that he referred to as bibliographic families consisting of derivations, sequels, adaptations, etc.). Vukadin (2014, p. 295) stated a Work can encompass "both the totality of transmedia intellectual content and its parts: The Matrix as well as, for example, the film The Matrix: Reloaded," but he meant this only with respect to the conceptual entity, as he went on to state that the FRBR Work's defined relationships would fail to "properly describe the essential whole/part nature of transmedia works" (p. 295-296).

The whole/part relationship that Vukadin emphasized is important for distinguishing FRBR Work from Superwork. Though a Superwork can be connected to its parts using various descriptive properties, the core of the relationship between a franchise-level Superwork and its various connected creative works is a "has part" or "is part of" relationship. The FRBR work-to-work relationship types are given as successor, supplement, complement, summarization, adaptation, transformation, and imitation (IFLA, 2006, p. 63), with no explicit whole-part relationships given for non-aggregates or multi-part works, neither of which adequately represents relationships in a multimedia franchise; similar shortcomings were noted by Vukadin (2014) and Tallerås et al. (2018). Thus, while the FRBR Work definition offers a level of ambiguity that allows one to argue it may represent a franchise, the intended use and practical relationships prescribed within FRBR indicate it is not the same type of entity. The types of relationships common between entities within a single franchise, such as significantly different intellectual content yet sharing the same fictional world and brand, do not appear to align with what the FRBR Work is intended to represent.

\section{Superwork versus FRBRoo Complex Work}

FRBR $_{\mathrm{OO}}$ is the object-oriented version of the FRBR family of conceptual models, and is intended to act as an alignment with CIDOC-CRM, and a formalization of FRBR concepts, enabling better adoption and implementation, both inside and outside the library world, using an ontological structure (Bekiari, Doerr, Le Boeuf, \& Riva, 2015). For this study, the main

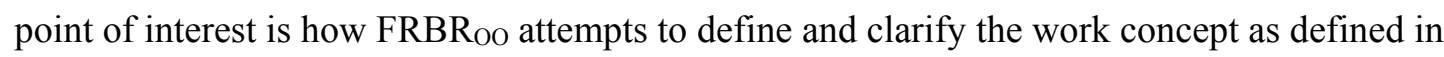

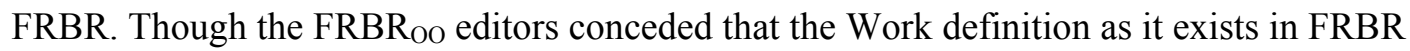
was open to interpretation, they retained the vague idea of Work as a superclass, while defining several other distinct work entities meant to represent the different interpretations of the original. These entities are the Work, Individual Work, Complex Work, Container Work, Aggregation Work, Serial Work, Publication Work, Performance Work, and Recording Work. As previous research (Lee et al., 2018; Vukadin, 2014; Tallerås et al., 2018; Kiryakos \& Sugimoto, 2019) has suggested that the FRBRoo Complex Work may be suitable for representing a Superwork-like entity, a comparison between Complex Work and Superwork as we have defined it is worth carrying out.

A Complex Work is defined as a class comprised of "works that have other works as members. The members of a Complex Work may constitute alternatives to, derivatives of, or self-contained components of other members of the same Complex Work" (Bekiari et al., 2015, p. 63). A Complex Work is associated with instances of the Work class through the has member property and allows for the "recognition of distinct sub-units, i.e. a complex work contained in a larger complex work" (p. 64). This definition is more closely related to the 
concept of a franchise-level Superwork, though, like the FRBR Work documentation, the examples fall short of explicitly stating this. Examples given of Complex Work (e.g., William Shakespeare's two-part play Henry IV, Walt Whitman's poetry collection Leaves of Grass), while composed of separate parts, may imply an intention to be consumed as a single unit that a franchise-level entity does not. Members of a Superwork may not share a direct part relationship, as is the case with Henry IV, and may not have any intentional collection effort, as with Leaves of Grass. Based on the documentation, Complex Work appears to have utility in representing distinct series or other sub-units within a franchise, rather than the entire franchise itself. These findings were echoed in Lee et al. (2018), stating that the Japanese TV animation series Mobile Suit Gundam may be conceptualized as a Complex Work, but it is more difficult to make this claim for the whole Gundam franchise to which the show belongs. Though Vukadin was more accepting of the use of Complex Work, she stopped short of saying it could be used as a franchise-level entity, instead stating that it provides support for the bibliographic modeling of transmedia relationships. Some support for Complex Work was also given by Tallerås et al. (2018) who viewed it as capable of collocating relevant works, but for fictional world levels of abstraction, defined as stories that "unfold based on (or at least referencing) a set of given characters, places, and events" (p. 901), FRBRoo "does not provide any sophisticated semantics for expressing such relationships" (p. 912).

\section{Superworks versus Subjects}

As Superwork is meant to represent a fairly high level of abstraction, a concern is whether a Superwork is in fact an entity, or is instead a type of subject. In attempting to define Superwork, Lee et al. (2018) faced a similar challenge and questioned whether what they referred to as a Superwork "was not better conceptualized as a kind of super-topic" (p. 6) similar to a Group 3 entity in FRBR. The issue with treating the Superwork as a subject was mainly a practical one: as subjects are typically the domain of various authorities (e.g., LCSH, NDLSH) "implementation would mean the insertion of countless new subject terms based on each franchise" (p. 6).

In addition to this implementation concern, a subject and a franchise-level entity may be thought of as implying two distinct concepts. We have stated that a Superwork is representative of a network of related works, and that it comprises some number of whole/part relationships. A subject, however, does not necessarily imply this type of connection, but rather describes the "aboutness" of a specific work. Thus, it may be possible for a work to be "about" a franchise without belonging to the network of resources that make up the Superwork. The two concepts are distinct, yet related: a Superwork is representative of some number of whole/part relationships that share a particular topic or subject, while a subject may be embodied by a group of related works that together form a franchise or Superwork.

In summary, both the relevant documentation and past research exploring FRBR Work and FRBR ${ }_{O O}$ Complex Work indicate that a franchise-level entity is not what was intended to be represented. FRBRoo provides useful clarification and classification of the FRBR Work concept, and Complex Work does have a role in the representation of some entities and relationships found in a multimedia franchise, but an entity that represents a franchise in its totality does not appear to be found in existing bibliographic models. Additionally, while Superwork is related to subject in that all the works a Superwork is connected to may share and embody particular topics, the attribution of a subject to a particular work does not imply the type of membership relation that the Superwork is conceived as representing. 


\section{EXISTING PORTRAYALS OF A FRANCHISE-LEVEL ENTITY}

If FRBR Work and FRBR ${ }_{0 O}$ Complex Work are not representative of franchise-level entities, it is worth examining what types of existing entities more closely resemble Superwork as it is envisioned in this paper. Lee et al. (2018) use the example of the Gundam franchise to illustrate a Superwork. Gundam refers to the Japanese science fiction / mecha multimedia franchise that began in 1979 with the animated TV series Mobile Suit Gundam, and has since spawned numerous other TV series, films, manga, novels, plastic figures, large real-life statues, etc. The Gundam franchise consists of numerous distinct series, for example Gundam Wing, Gundam SEED, Gundam Unicorn, which differ based on timelines, universes, characters, etc., but have the common theme of the featuring giant robots referred to as Gundam. In their conceptualization of the Gundam franchise (see Lee et al., 2018, Figure 2) the Gundam Superwork entity uses an includes relationship to connect the Superwork to various components such as a TV series, film, original video animation, character, music soundtrack, plastic model kit, etc. The Superwork entity encompassing a variety of resource types from different series within the Gundam franchise is a clearly distinct conceptual entity from the examples of Complex Work discussed earlier. In this example, Complex Work appears to be suited to represent the series within the franchise (e.g., Gundam Wing, Gundam SEED) and any recursive Complex Work entities that belong to them, rather than an entity representing the franchise as a whole. Using this conceptualization, they sought to define other properties of the entity, stating that a Superwork is a type of aggregate encompassing multiple related works and formats, containing works by several authors aggregated around a theme or group of related themes. These conceptualizations and characteristics are in line with the way Superwork is defined in this paper.

\section{User-created Superwork Entities in Existing Literature}

Much of the discussion on Superwork and related entities has been from researchers or other domain experts applying their knowledge to bibliographic organization, with few extant userbased research. In addressing this, Tallerås et al. (2018) asked a group of users to conceptualize and map out relationships based on three related resources sourced from two different works. While the works in question (Peer Gynt and Romeo and Juliet) are smaller in scale than multimedia franchises like Star Wars or Gundam, their findings are applicable: the authors found that when given resources representing a book, movie, and movie soundtrack and asked to conceptualize and map the resources, the majority chose to create a new "central node" entity with which to connect the resources to (see Figure 7 and 8 in Tallerås et al., 2018, p. 906-907). Users were also able to apply attributes to each resource, with the results showing that users "tend to have persons of responsibility related to the central node but provide other attributes at the document level, such as the date of publication" (p. 909). This implies that the central node cannot be interpreted as a FRBR Work, as the person of responsibility (e.g., William Shakespeare) would not be responsible for the music soundtrack of a film adaptation of Romeo and Juliet. Based on this, the authors stated that the central node may be interpreted as an "abstract collocating device" and that creators may be linked to the central node "because they are responsible for the originator works of the bibliographic families," suggesting that the node "more resembles a superwork entity" (p. 911).

It should be noted here that the authors' use of the term Superwork is closely related to FRBRoo's Complex Work and that another layer of abstraction related to the content of the documents, the "fictional world," is given. The fictional world level of abstraction does not 
appear to be a work itself, but rather the common thread that the works are being collocated around. This interpretation closely resembles the franchise-level Superwork as discussed in this paper. The user-generated central node as a representation of a fictional world level of

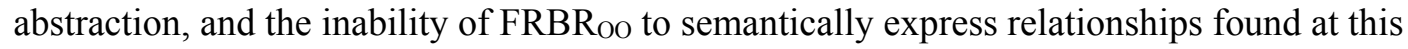
level, are important findings, as they show that users will create a central conceptual entity with which to connect distinct, yet related resources, and that the central node will only be associated with attributes that can be applied to each of the connected resource in some way, such as the original story creator.

Though the works in question differ from the large multimedia franchises that are the subject of this paper, it is reasonable to assume that the majority of users would likewise create a similar entity if given resources related to a franchise and its distinct series such as Gundam. This assumption is based on the observation that each of the distinct series within the Gundam franchise more closely resembles Romeo and Juliet than the franchise as a whole, and thus an even higher level of abstraction would need similar collocation efforts. This assumption is further supported in the way that users create collocation entities for multimedia franchises on the Web.

\section{User-created Superwork Entities on the Web}

While the study of Tallerås et al. (2018) showed that users will create central nodes when asked to conceptualize and map related resources, there remained the question of whether a similar practice occurs when unprompted by researchers. In analyzing the FilePlanet website and examining user-made modifications for the PC game Doom, McDonough et al. (2010) wrote that a Superwork entity may support users' preferred mode of searching (p. 31), as the users were already collocating works by themselves. However, it was not clear whether these collocations, based on game engines, would represent the same type of Superwork entity discussed here. Wikipedia, however, provides a larger case study, and does seem to support the idea that users will create Superwork-like entities for multimedia franchises.

The List of Multimedia Franchises article on Wikipedia ${ }^{1}$ is a collection of media franchises which, to qualify for inclusion in the article, is defined as a franchise having "works in at least three forms of media, and must have two or more separate works in at least two of those forms of media" (paragraph 2). Prominent examples include Blade Runner, Jurassic Park, Garfield, as well as franchises mentioned earlier like Pokémon, Star Wars, Ghost in the Shell, Middle-earth, Marvel Cinematic Universe, and Gundam. An important trait common to most of these is the existence of a single Wikipedia article that represents the whole franchise and not simply a list of separate articles for each media format, though these format-specific articles do exist and are typically linked to from the franchise article. For example, the articles for Blade Runner, Godzilla, and The Matrix have the term franchise directly in their page titles, with others like Jurassic Park forgoing this title addition but stating in the opening sentence that the article represents the total media franchise. Some examples such as Harry Potter do not have a single franchise-level article but rather large articles for each medium (e.g., film series and novel series). Importantly, however, these medium-specific articles do discuss the entire franchise to some degree: the Harry Potter article is explicitly about the novels, but also features a substantial adaptations section that, in addition to linking to separate articles about the film series, video games, theme parks, etc., also describes each of them in some detail. Interestingly, the Harry Potter (film series) article

\footnotetext{
${ }^{1}$ https://en.wikipedia.org/wiki/List_of_multimedia_franchises
} 


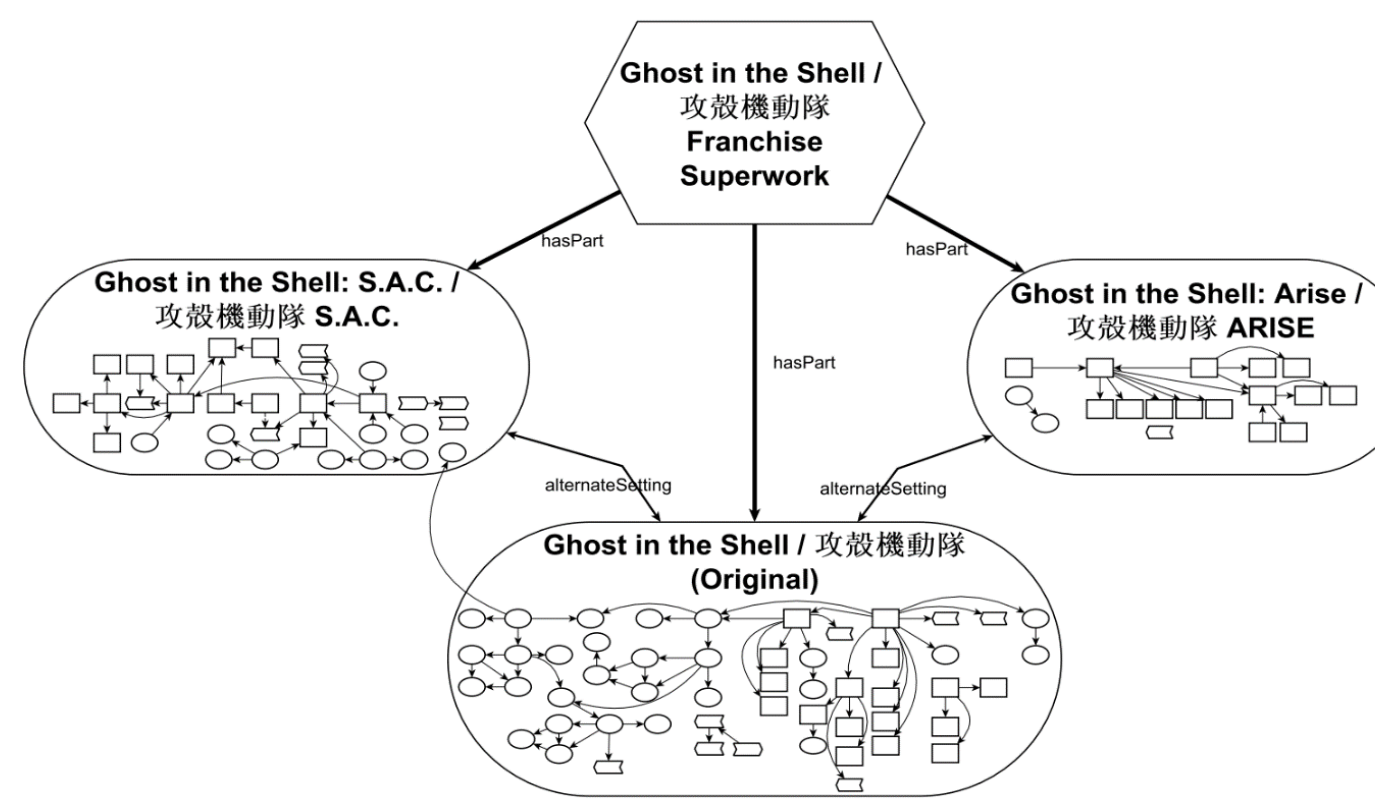

Figure 1. Abstract overview of mapped entities and relationships between resources mentioned Wikipedia articles for the Ghost in the Shell franchise, grouped by series.

lacks these descriptions about adaptations, and so only the article for the novel series can be said to resemble the more explicit franchise article examples. The reason for this may be because the novels were the original medium, though further investigation is needed to make this claim with certainty. In both cases, the franchise-level articles can be said to be a representation of a conceptual Superwork entity. Similar to the finding of selective attribution application by users in the study of Tallerås et al. (2018) discussed earlier, these articles typically feature attributes relevant to the whole franchise rather than a specific medium (e.g., original story creator, first instantiation, unifying subjects or themes). They also connect different franchises based on abstract concepts such as crossovers or inspirations. A franchiselevel entity is vital here, as works may be influenced by the themes, world, etc. present across an entire franchise and not just a single instance within that franchise, thus requiring franchise-level entities for an accurate portrayal of this relationship.

Lastly, these Wikipedia entries both describe and link to a variety of articles for related series, media types, and other derivatives. If the franchise article represents a Superwork, then this network of connected articles represents the membership network of instances that form the Superwork. Though the existence of franchise-level pages is arguably sufficient to claim that users on the Web do create conceptual collocating entities for media franchises, an analysis of the franchise page and its relationships to other articles would be helpful in revealing the relationship types that exist between different entities, the entities between which certain relationships hold, and the boundaries of a Superwork by determining what types of media are included in certain pages. In attempting to visualize these relationships, a mapping of Wikipedia entities for the Ghost in the Shell / 攻殼機動隊 franchise was performed, an abstract overview of which is shown in Figure 1.

Though the main Ghost in the Shell franchise article appears to be separated by media formats, these distinctions usually refer to the original medium that a new series appeared in and not a listing of all Ghost in the Shell works that exist in that format. For example, Ghost in the Shell: The New Movie, an animated film, is not listed under Films but instead Television, as it is part of the Ghost in the Shell: Arise series which originated as a TV series. 

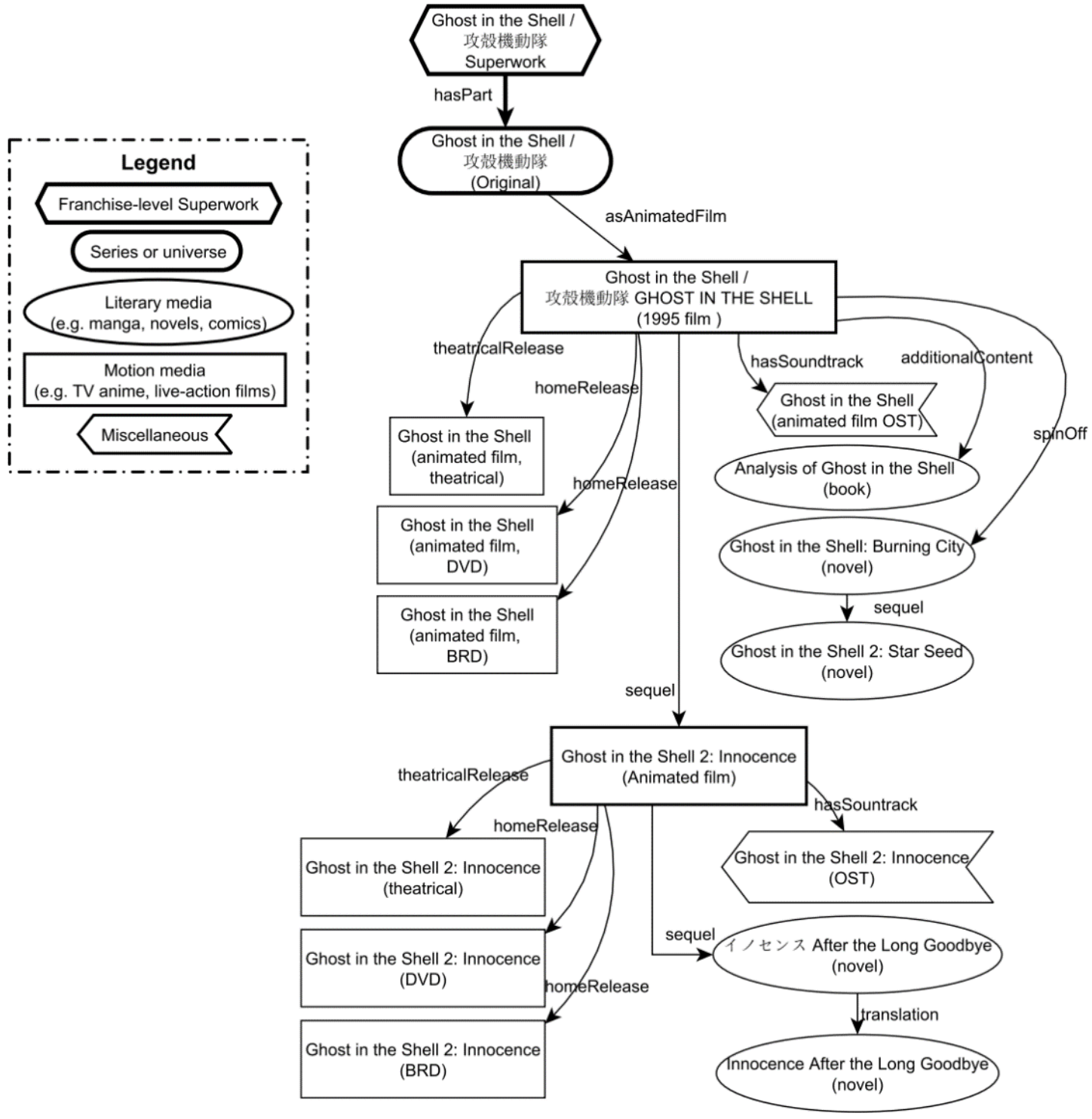

Figure 2. Excerpt from the Wikipedia mapping showing the Ghost in the Shell (Original) animated film resource and connected entities sourced from Wikipedia data

Therefore, the organization of the content of the Ghost in the Shell articles, and thus the mapping, was done mainly by series. The groupings in Figure 1 show the entities representing the three series "Original", "Stand Alone Complex" (S.A.C.) and "Arise," and their connection to the Superwork entity representing the central franchise-level Wikipedia article.

As previously stated, the purpose of performing a mapping beyond the franchise page is to examine the relationships found among different Wikipedia pages related to the franchise. Figure 2 shows an excerpt from the Wikipedia mapping showing entities belonging to the Ghost in the Shell (Original) series. This series began the franchise and was originally conceived as a manga, though it exists in multiple adaptations, of which the animated film version is shown in the figure. While the franchise-level Wikipedia article does have a link to both the Ghost in the Shell (1995 film) and Ghost in the Shell 2: Innocence entities, the various other entities linked to and mentioned in those articles (e.g., novel sequels, unique released, and soundtracks) are only featured in each respective film's article. This reflects the findings by Tallerås et al. (2018, p. 906-907) that users may not always connect every 
resource to the central node, but instead connect a resource such as the music soundtrack to the movie resource itself, rather than to the central node. Though this finding is not surprising, as linking every possible media instance related to a franchise would result in Wikipedia franchise articles being overly lengthy, it does illustrate how users will intelligently collocate certain materials belonging to a single series, even if they also create a central node that is capable of having all possible resources connected to it.

\section{DISCUSSION}

A significant portion of the research presented in this paper was focused on the clarification of what a franchise-level entity is and if any entities in existing bibliographic models were intended to represent such an entity. Based on official documentation and related research, we

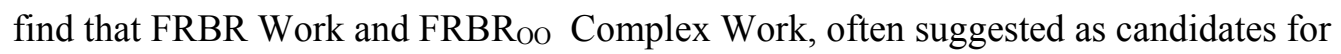
Superwork, are not capable of representing a whole multimedia franchise, though their roles have been made clearer. While Superwork as discussed in this paper may be used for franchise-level representation, Complex Work appears suitable for representing the distinct series that exist within the franchise, for example the three series entities shown in Figure 1. Additionally, past research on transmedia works reveals that the term Superwork may be used to refer to a series rather than a franchise, making the comparison to Complex Work more straightforward. Thus, for greater clarity in future papers, it may be beneficial for authors to maintain the concept of Superwork for the franchise-level entity as discussed in this paper, but utilize an alternative term that more explicitly implies a franchise connection based on a higher level of abstraction such as universe (Lee, Tennis, Clarke, \& Carpenter, 2013) or fictional world (Tallerås et al., 2018). Though these alternative terms can capture the fluid interpretations of Superwork or Complex Work (e.g., "fictional worlds typically are developed through stories referencing the same characters, places, or events within or across authorships" (Tallerås et al., 2018, p. 898) avoidance of the term Superwork in referring to an entity that differs from Complex Work (e.g., by using a term such as franchise work) may be helpful in providing clarity in future research.

In addition to differentiating Superwork from existing entities, this research has examined how entities that represent franchises are currently portrayed in Wikipedia, and whether the portrayal is consistent with Superwork-as-a-franchise as conceptualized in this and previous studies. The existence of Wikipedia articles for entire franchises suggest that users will create conceptual entities with which to collocate a variety of related works and use levels of description that are typically agnostic of single media types within that franchise. This enables features such as description of themes or topics that are present across the whole franchise, and the connection of one franchise to another for the purposes of describing inspirations or crossover works that are accurately made between franchises rather than between single media types from either franchise. There are other descriptions that users seemingly prefer to discuss in the context of an entire franchise rather than specific instances. Importantly, the collocation of various media types, and the connection to other franchises via relationships such as inspirations or crossovers are vital tools for improving search and retrieval.

\section{Boundaries}

Based on existing Wikipedia franchise articles, the boundaries on the types of media formats that warrant a direct connection to the Superwork appear to vary depending on the franchise and how important or prominent a given medium is to that franchise. Using the example of 
small statues or figures, any mention of them are absent from the Ghost in the Shell franchise article, though they are briefly mentioned alongside other merchandise in the "Related Media - Other" section of the Ghost in the Shell: S.A.C. page. ${ }^{2}$ In contrast, "Gunpla" or "Gundam Plastic Models" are a significant aspect of the Gundam franchise, resulting in both its own Wikipedia page ${ }^{3}$ and a link to this page from the central Gundam franchise article. The variation in the importance of different media types for a franchise implies that rather than prescribing boundaries in this area, it is better to leave such definitions to the communities themselves.

\section{Challenges and Future Research}

Though this study has focused on a group of issues meant to differentiate a multimedia franchise from existing bibliographic entities, other challenges emerged at various stages of the research. Limiting the existing entity analysis to FRBR Work and FRBR OO $_{\text {Complex }}$ Work is not a significant limitation, as these have been the most often suggested entities in the literature for representing concepts presented here. However, newer models, that is Library of Congress' BIBFRAME and IFLA's Library Reference Model (LRM), should be subjected to the same analysis in future work. BIBFRAME defines its Work entity as "the highest level of abstraction" and as "the conceptual essence of the cataloged resource: authors, languages, and what it is about (subjects)" (Library of Congress, 2016). Because the franchise-level entity is arguably a level of abstraction above the essence of a catalogued resource (as typically, single creative works and not franchises are the catalogued resource), it appears that this would not be suitable for representing a franchise, though a definitive answer awaits a more thorough analysis. LRM also includes conceptual entities which may be able to represent the franchise as a subject but may fail to portray the part of relationships that Superwork members have.

The precise nature of the relationship between a Superwork and its members or series was not addressed in this paper. Though the relationship between Superwork and series in our Wikipedia mapping was represented as a generic "hasPart" property, this may imply a specific type of multi-part work or series depending on the bibliographic model in question. While a franchise and its connected works do seem to have a whole/part relationship, there may be more appropriate terms to indicate membership into a franchise, such as hasMember/isMemberOf, or a generic includes property as seen in Lee et al. (2018). The difference between these properties and the types of relationships they imply, while subtle, is significant, and any future research that examines the relationship between a franchise and its members should attempt to clarify this.

An issue with the term franchise or multimedia franchise is that it may not have a universally accepted definition. Though this is not a significant issue when discussing the franchise-level entity conceptually, the lack of an accepted definition for multimedia franchise can lead to unforeseen complications similar to those faced by Lee et al. (2018), who encountered difficulties in determining whether or not crossover works such as the Kingdom Hearts series, which is primarily a series of video games featuring characters and worlds from both the Final Fantasy and Disney franchises, was itself a Superwork. Interestingly, the List of Multimedia Franchises Wikipedia article discussed earlier does attempt to define a multimedia franchise as having "works in at least three forms of media and must have two or more separate works in at least two of those forms of media" (para. 2). This definition, however, is specified as a criterion for determining whether a franchise qualifies for entry in

\footnotetext{
${ }^{2} \mathrm{https} / / /$ en.wikipedia.org/wiki/Ghost_in_the_Shell:_Stand_Alone_Complex\#Other ${ }^{3}$ https://en.wikipedia.org/wiki/Gundam_model
} 
the list, and so a definition without this function may differ. The background section of this paper also illustrates how the use of the term transmedia can vary, and so, attempts at defining a multimedia franchise may encounter issues when compared to closely related terms. Still, attempts at defining a multimedia franchise or a more thorough examination of the multitude of existing definitions may be useful for more plainly delineating ideas presented in this research.

An issue related to boundaries that this paper did not address is the role of fan-created works in the relationship network of a franchise. Similar to other boundary issues, this is best left to users in the various communities, as the importance of fan works can vary between franchises. Referring to the Wikipedia examples discussed earlier, the Ghost in the Shell article does not contain discussion on or links to any fan works, while the Cultural Impact section of the Harry Potter article both discusses fan works and links to a separate Harry Potter Fandom article (see: https://en.wikipedia.org/wiki/Harry_Potter_fandom). The challenge here is in allowing a level of fluidity in the definition of a franchise-level Superwork, rather than implying a kind of canonicity (Smiraglia, 2007). Fan works may overly broaden the boundaries. A more thorough investigation of this issue should be performed in the future.

Though we are satisfied that a franchise-level entity and FRBR ${ }_{\mathrm{OO}}$ Complex Work are adequately differentiated, it may be beneficial to investigate the role of Complex Work in representing single series or universes mentioned previously. Conceptually, Complex Work appears to be able to represent a series entity, though the restrictions on relationship types that a Complex Work can participate in may hinder this level of representation, just as they did for the franchise-level concept. An analysis of series-level articles rather than franchise-level ones may provide more insight into the types of relationships users create here, and whether Complex Work is suited for portraying them.

A user study similar to that performed by Tallerås et al. (2018) may be helpful in clarifying some of the questions raised in our Wikipedia analysis, such as how to determine the importance of certain media types or merchandise, such as soundtracks and figures, to a franchise-level article. Another interesting question is why some franchises such as Blade Runner or Jurassic Park are given explicit franchise articles, whereas other articles are more implicit in indicating a franchise, such as the main Harry Potter article which is clearly for the novel series despite containing data for and linking to other media formats and adaptations in the franchise. This may be a result of Wikipedia's editing or other structural decisions, and not a conceptualization choice made by users. However, as other franchises with more dominant media types exhibit similar behavior, the question remains unanswered.

\section{CONCLUSION}

Though the scope and conclusions of this research cannot be considered exhaustive in resolving issues related to the portrayal of multimedia franchises using existing bibliographic models and their entities, clarifications on specific entities, often suggested for portraying multimedia franchises, has been presented. While FRBR Work and FRBR OO $_{\text {Complex Work }}$ certainly have roles in the portrayal of certain entities and relationships within a franchise, there is room for a singular entity to represent a multimedia franchise in its entirety. This entity not only enables the type of collocation that users create and prefer on the Web, but also allows for new relationships to be expressed, like crossover works or inspirations, thus having a direct impact on search and retrieval. An analysis of the user-created relationship networks that exist on Wikipedia provide some insights into how users not only create these 
collocations, but also establish relationships between entities in the network of resources that form a multimedia franchise. These relationships are varied and complex, and provide useful guidance on how any formal bibliographic entity that represents a franchise and its relationship network should be created.

In addition to this study, other projects currently underway involve the connection of related entities across different media types present in the Japanese governmental Media Arts Database, ${ }^{4}$ and other similar international collaborative work focused on linking related works. User-created relationships between resources, both within a single franchise and between distinct franchises, have helped inform how these projects can better meet user needs in the way they describe and link related works, and help institutions better accommodate the growing transmedia phenomenon.

\section{REFERENCES}

Bekiari, C., Doerr, M., Le Boeuf, P., \& Riva, P. (Eds.) (2015). FRBR object-oriented definition and mapping from FRBR $R_{E R}, F R A D$ and FRSAD (version 2.4). International Working group on FRBR and CIDOC CRM harmonization.

Bennett, R., Lavoie, B. F., \& O'Neill, E. T. (2003). The concept of a work in WorldCat: An application of FRBR. Library Collections, Acquisition and Technical Services, 27(1), 45-59. https://doi.org/10.1016/S1464-9055(02)00306-8

Branch, F., Arias, T., Kennah, J., Phillips, R., Windleharth, T., \& Lee, J. H. (2017).

Representing transmedia fictional worlds through ontology. Journal of the Association for Information Science and Technology, 68(12), 2771-2782. https://doi.org/10.1002/asi.23886

Carlyle, A. (1996). Ordering author and work records: An evaluation of collocation in online catalog displays. Journal of the American Society for Information Science, 47(7), 538554.

Carlyle, A. (1999). User categorisation of works: Toward improved organisation of online catalogue displays. Journal of Documentation, 55(2), 184-208. https://doi.org/10.1108/EUM0000000007143

Dena, C. (2009). Transmedia practice: Theorising the practice of expressing a fictional world across distinct media and environments (Unpublished doctoral dissertation). School of Letters, Art and Media, University of Sydney, Australia. Retrieved from http://cirettransdisciplinarity.org/biblio/biblio_pdf/Christy_DeanTransm.pdf

Ercegovac, Z. (2006). Multiple-version resources in digital libraries: Towards user-centered displays. Journal of the American Society for Information Science and Technology, 57(8), 1023-1032. https://doi.org/10.1002/asi.20367

Giovagnoli, M. (2011). Transmedia storytelling: Imagery, shapes and techniques. ETC Press.

IFLA Study Group on the Functional Requirements for Bibliographic Records. (2006).

Functional Requirements for Bibliographic Records. Retrieved from https://www.ifla.org/publications/functional-requirements-for-bibliographic-records

Jenkins, H. (2003). Transmedia storytelling. MIT Technology Review, 28. Retrieved from https://www.technologyreview.com/s/401760/transmedia-storytelling

Jenkins, H. (2006). Convergence culture: Where old and new media collide. New York: New York University Press.

\footnotetext{
${ }^{4}$ https://mediaarts-db.bunka.go.jp/
} 
Jett, J., Sacchi, S., Lee, J. H., \& Clarke, R. I. (2016). A conceptual model for video games and interactive media. Journal of the Association for Information Science and Technology, 67(3), 505-517. https://doi.org/10.1002/asi.23409

Kiryakos, S., \& Sugimoto, S. (2015). A linked data model to aggregate serialized manga from multiple data providers. In Proceedings of the 17th International Conference on AsiaPacific Digital Libraries (pp. 120-131). Berlin, Heidelberg: Springer-Verlag. https://doi.org/10.1007/978-3-319-27974-9_12

Kiryakos, S., \& Sugimoto, S. (2019). Building a bibliographic hierarchy for manga through the aggregation of institutional and hobbyist descriptions. Journal of Documentation, 75(2), 287-313. https://doi.org/10.1108/JD-06-2018-0089

Kiryakos, S., Sugimoto, S., Lee, J.H., Jett, J., Cheng, Y., \& Downie, J.S. (2017). Towards a conceptual framework for Superworks. In Proceedings of the 7th Conference of Japanese Association for Digital Humanities, September 11-12, 2017 (pp.47-49). Japanese Association for Digital Humanities.

Kiryakos, S., Sugimoto, S., Nagamori, M., \& Mihara, T. (2016). Aggregating metadata from heterogeneous pop culture resources on the Web. In DC-2016 Proceedings:

International Conference on Dublin Core and Metadata Applications (pp. 65-74). Retrieved from http://dcpapers.dublincore.org/pubs/article/view/3824

Leazer, G. H., \& Furner, J. (1999). Topological indices of textual identity networks. In Proceedings of the 62nd Annual Meeting of the American Society for Information Science (pp. 345-358). Medford, NJ: Information Today.

Leazer, G. H., \& Smiraglia, R. P. (1999). Bibliographic families in the library catalog: A qualitative analysis and grounded theory. Library Resources and Technical Services, 43(4), 191-207.

Lee, J. H., Jett, J., Cho, H., Windleharth, T., Disher, T., Kiryakos, S., \& Sugimoto, S. (2018). Reconceptualizing superwork for improved access to popular cultural objects.

Proceedings of the Association for Information Science and Technology, 55(1), 274281. https://doi.org/10.1002/pra2.2018.14505501030

Lee, J. H., Shim, Y., \& Jett, J. (2015). Analyzing user requests for anime recommendations. In Proceedings of the 15th ACM/IEEE-CE on Joint Conference on Digital Libraries JCDL '15 (pp. 269-270). New York: ACM Press. https://doi.org/10.1145/2756406.2756969

Lee, J. H., Tennis, J. T., Clarke, R. I., \& Carpenter, M. (2013). Developing a video game metadata schema for the Seattle Interactive Media Museum. International Journal on Digital Libraries, 13(2), 105-117. https://doi.org/10.1007/s00799-013-0103-x

Lee, S., \& Jacob, E. K. (2011). An integrated approach to metadata interoperability. Library Resources \& Technical Services, 55(1), 17-32. https://doi.org/10.5860/1rts.55n1.17

Library of Congress. (2016). Overview of the BIBFRAME 2.0 model. Retrieved from https://www.loc.gov/bibframe/docs/bibframe2-model.html

McDonough, J., Kirschenbaum, M., Reside, D., Fraistat, N., \& Jerz, D. (2010). Twisty little passages almost all alike: Applying the FRBR model to a classic computer game. DHQ: Digital Humanities Quarterly, 4(2). Retrieved from http://digitalhumanities.org:8081/dhq/vol/4/2/000089/000089.html

Merčun, T., Žumer, M., \& Aalberg, T. (2012). Presenting and exploring the complexity of bibliographic relationships. In H.H. Chen \& G. Chowdhury (Eds), The outreach of digital libraries: A globalized resource network, ICADL 2012 (pp. 63-66). Berlin: Springer. https://doi.org/10.1007/978-3-642-34752-8_8 
Merčun, T., Žumer, M., \& Aalberg, T. (2017). Presenting bibliographic families using information visualization: Evaluation of FRBR-based prototype and hierarchical visualizations. Journal of the Association for Information Science and Technology, 68(2), 392-411. https://doi.org/10.1002/asi.23659

Niu, J. (2013). Hierarchical relationships in the bibliographic universe. Cataloging and Classification Quarterly, 51(5), 473-490. https://doi.org/10.1080/01639374.2012.749969

Smiraglia, R. P. (2007). Bibliographic families and superworks. In Taylor, A. G. (Ed.), Understanding FRBR: What it is and how it will affect our retrieval tools (pp. 73-86). Westport, CT: Libraries Unlimited.

Smiraglia, R. P., \& Leazer, G. H. (1999). Derivative bibliographic relationships: the work relationship in a global bibliographic database. Journal of the American Society for Information Science, 50(6), 493-504.

Smiraglia, R. P., Riva, P., \& Žumer, M. (2014). The FRBR family of conceptual models: Toward a linked bibliographic future. London: Routledge.

Svenonius, E. (2000). The intellectual foundation of information organization. Cambridge, MA: MIT Press.

Tallerås, K., Dahl, J. H. B., \& Pharo, N. (2018). User conceptualizations of derivative relationships in the bibliographic universe. Journal of Documentation, 74(4), 894-916. https://doi.org/10.1108/JD-10-2017-0139

Teixeira, M. (2012). Contributions to FRBRization: The library catalogue in a new digital environment: How FRBR can help produce better display in OPACs. Retrieved from https://www.academia.edu/215914/Contributions_to_FRBRization_The_library_catalo gue_in_a_new_digital_environment_how_FRBR_can_help_produce_better_displays_i n_OPACs

Vukadin, A. (2014). Bits and pieces of information: Bibliographic modeling of transmedia. Cataloging and Classification Quarterly, 52(3), 285-302. https://doi.org/10.1080/01639374.2013.879976

Wilson, P. (1968). Two kinds of power: An essay on bibliographical control. Berkeley: University of California Press.

Zhang, Y., \& Salaba, A. (2012). What do users tell us about FRBR-based catalogs?

Cataloging and Classification Quarterly, 50(5-7), 705-723.

https://doi.org/10.1080/01639374.2012.682000 